\title{
Analysis and Simulation of the ITER-Type NbTi Bus BarIII With the THELMA Code
}

\author{
F. Bellina, Member, IEEE, P. L. Ribani, M. Bagnasco, L. Muzzi, E. Salpietro, L. Savoldi Richard, and R. Zanino
}

\begin{abstract}
Bus Bar III (BBIII) is a curvilinear NbTi full-size ITER-type multistrand superconducting cable $7 \mathrm{~m}$ long, tested in 2004 at Forschungszentrum Karlsruhe, Germany. The sample was cooled with a forced helium flow and was equipped with many electromagnetic and thermal-hydraulic sensors, to acquire as wide as possible an experimental database, aimed at getting a benchmark for the validation of the THELMA code, a computer tool for the analysis of CICC prototypes and magnets, recently developed and presently under validation. This code can compute the current distribution among the macrostrands used to represent the cable, taking into account their superconducting behavior, as well as the temperature and the magnetic field distribution. The paper deals with the application of THELMA to some tests on BBIII useful for the electromagnetic model validation and presents in detail the analysis setting-up, the results, its critical aspects and limits. A comparison is shown between the measured and the computed waveforms of the electromagnetic data.
\end{abstract}

Index Terms-Fusion reactors, ITER, NbTi, superconducting magnets.

\section{INTRODUCTION}

A TOOL TO simulate the behavior of magnets made with multistrand superconducting cable-in-conduit conductor (CICC) can be very helpful for a better comprehension of the experimental results and to anticipate the performances of future full-size magnets. A number of computer codes have been produced, which can model in detail some of the aspects of the magnet behavior or can analyze it in a particular regime [1], [2]. Among these codes, THELMA has been developed with the target to analyze the CICC behavior taking into account the effect of the electrical joints and terminations and the thermal-hydraulic aspects [3]. The paper discusses the main results of the application of THELMA to the BBIII experiment, carried out in 2004 in the TOSKA facility at Forschungszentrum Karlsruhe, Germany [4]. The collected experimental data have been used for the validation of the THELMA code, presented in this paper, and constitute a database to test the current identification methods based on the magnetic field measurements.

Manuscript received September 20, 2005. This work was supported in part by EFDA task TW3-TMSC-CODES through ENEA contract, and by MIUR.

F. Bellina is with Università di Udine, Dipartimento di Ingegneria Elettrica, Gestionale e Meccanica, I 33100 Udine, Italy (e-mail: Fabrizio.bellina@uniud. it).

P. L. Ribani is with Università di Bologna, Dipartimento di Ingegneria Elettrica, I-40136 Bologna, Italy (e-mail: pierluigi.ribani@mail.ing.unibo.it).

M. Bagnasco, L. Savoldi Richard, and R. Zanino are with Dipartimento di Energetica, Politecnico, I-10129 Torino, Italy (e-mail: maurizio.bagnasco@ polito.it; laura.savoldi@polito.it; roberto.zanino@polito.it).

L. Muzzi is with ENEA, Centro Ricerche Frascati, I-00044 Frascati, Italy (e-mail: muzzi@frascati.enea.it).

E. Salpietro is with EFDA-CSU, D-85748 Garching, Germany (e-mail: ettore. salpietro@tech.efda.org).

Digital Object Identifier 10.1109/TASC.2005.864251
In all the BBIII tests, the conductor behavior was driven with a proper resistive heating of the cooling helium or the cable jacket, and by feeding the cable with suitable current waveforms. The first set of tests were devoted to the calibration of the magnetic field probes. To the purpose, a uniform current distribution was obtained among the strands by keeping the cable temperature at $77 \mathrm{~K}$ : at this temperature the uneven electrical contact resistances at the cable joints are quite negligible with respect to the strand uniform longitudinal resistance. The second set of tests were carried out to study specifically the electromagnetic behavior in the linear regime, in the absence of any resistive strand transition. The third set of tests were current-sharing pulses. The details of BBIII, of its diagnostic equipment, as well as the experimental campaign, are described in [4].

\section{THELMA VALIDATION SETTING-UP}

To validate THELMA, the data measured during a real experiment on a prototype are to be reproduced by the code as close as possible, through a set of representative models based on the geometrical data, the material properties, the applied currents and heating power. The THELMA electromagnetic (EM) model of a CICC can be very detailed, since the code allows the cable to be represented as a set of bundles made of different strands/macrostrands. However, the more detailed the cable description in the model is, the larger is the number of necessary input data, as well as the corresponding computational cost of the simulation. As regards BBIII, in addition to the geometrical data, a number of parameters, like some contact resistances could be found or reliably extrapolated from other experiments on similar types of cable [5]. Some other parameters, like the contact resistances in the joint, were not known, but their value could be determined by fitting the experimental and the computed data. Unfortunately, no exhaustive database of the BBIII strand critical properties is available, therefore the THELMA validation on BBIII was focused on the EM aspects.

\section{BBIII EM MODELS}

Two THELMA EM models of the BBIII cable have been considered: with 6 or 24 macrostrands. In the first model, each macrostrand corresponds to a cable petal (192 strands) while, in the second, it corresponds to a subpetal (48 strands). Both models include the inlet and outlet joint copper saddles [Fig. 1(a)], represented as a lumped network which is automatically generated. In addition, linear resistors corresponding to the electrical contacts are automatically considered wherever the macrostrands are in geometrical contact with the saddles [6]. The model takes into account also the removal of the petal wrapping in the contact surface of the saddle. Each interface between the saddle and the rest of the circuit is assumed to be 

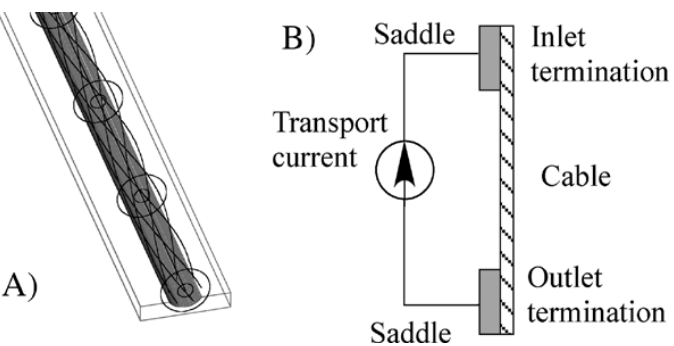

Fig. 1. (A) Detail of the EM model of the joint. The 6 macrostrands middle lines are reported together with the saddle and cable outlines. (B) Sketch of the EM model circuit.

an equipotential surface, and the whole busbar is supposed to be fed with an impressed known current waveform [Fig. 1(b)]. The part of cable between the two joints is described by a distributed parameter model, in which any macrostrand is characterized by its own current and magnetic flux density, that are functions of time and the longitudinal coordinate along the cable [7], [8]. In each macrostrand cross-section, both the current density and the magnetic flux density are supposed to be uniform. The models include the magnetic coupling between different zones of the cable.

\section{ANalysis of the CAlibration Pulses @ 77 K}

For these tests, a trapezoidal current with a plateau value of $1 \mathrm{kA}$ and different ramp rates was applied. In all the tests, the real cable showed completely resistive behavior. The magnetic field values measured in these conditions have been considered as calibration constants, to be used to compute the signal unbalances due to an uneven current distribution among the strands at low temperature. In our simulations, among all available magnetic field probes, we selected probes heads HP5 and HP6, located approximately at the cable middle length, because many of their actual geometrical data have been measured with a high accuracy [9]. This revealed to be a key aspect of the analysis, since the measured individual probe angular misalignment errors (up to $3.8^{\circ}$ ), as well as the effect of the different thickness of the jacket walls (up to $0.7 \mathrm{~mm}$ ) had to be taken into account to fit the experimental data. Fig. 2 compares the field values measured during the plateau (when all the inductive effects are quite negligible) with those computed considering the geometrically corrected probe data. Due to the cable resistive behavior, this field computation was performed at the steady-state. As it can be seen, as regards the tangential field probes, the difference between the computed and the measured values is of the same magnitude as the difference between the values measured in pulses with different ramp rates. The radial field probes measured signals about one magnitude lower, due to their location and orientation. For these probes, a large relative (but small absolute) error is still present. To understand the reason of these small residual field errors, we compared them with the effect of other possible sources of geometrical error that could be present during the manufacture and installation of BBIII. As a first case, we considered the effect of a rigid rotation of both the probe heads of $0.3^{\circ}$ about the cable. This rotation may correspond to a parallelism shape tolerance of $0.26 \mathrm{~mm}$ for the two jacket upper and lower surfaces, with respect to the busbar middle plane.
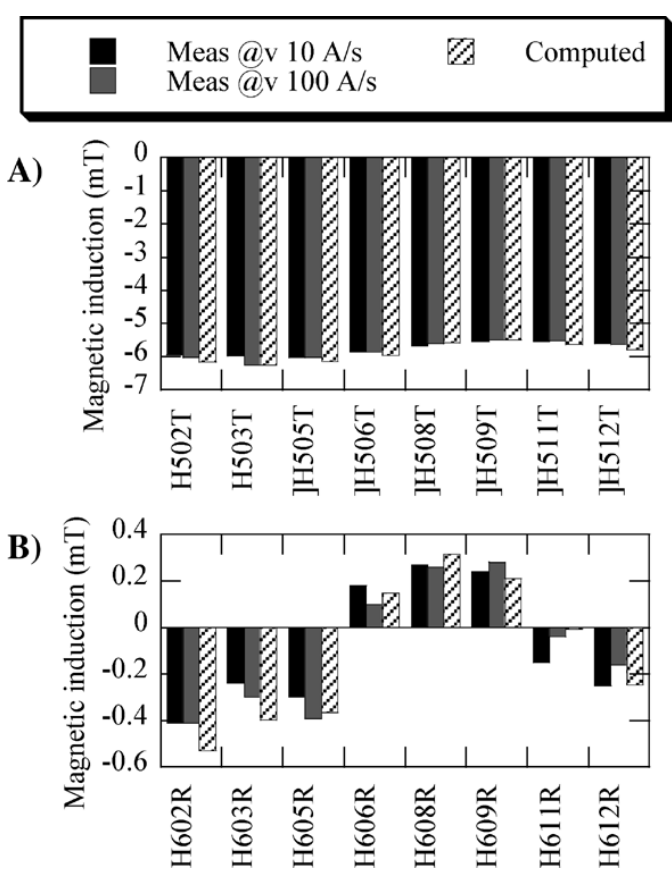

Fig. 2. Pulses @ $77 \mathrm{~K}$ comparison between the measured field values and the computed values during the plateau @ 1 kA. (A) Tangential HP5 field probes. (B) Radial field HP6 probes.

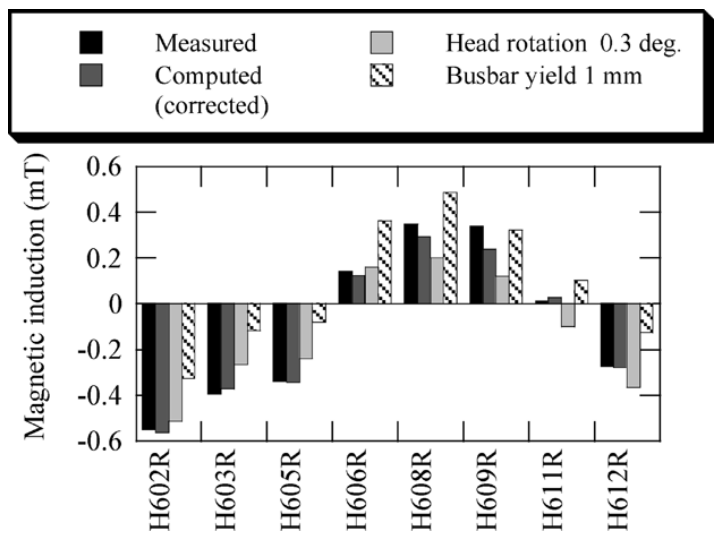

Fig. 3. Pulses @ $77 \mathrm{~K}$ and $1 \mathrm{kA}$ : Effect of an angular rotation of HP6 probe by $0.3^{\circ}$ and a vertical yield of $1 \mathrm{~mm}$ of the central part of the busbar.

Then we considered also the effects of small geometrical deformations of the cable middle line, assuming a vertical yield of $1 \mathrm{~mm}$ of the central part of the busbar. In this case the two end parts of the cable remain undistorted, and the probes keep their relative position with respect to the deformed busbar. The results of this analysis are reported in Fig. 3, and they show that these small applied rotations and displacements can give field errors even larger than the difference between measured and computed values. Since these rotations/displacements are well within the typical manufacturing and installation tolerances for this type of objects, we conclude that they could be a possible reason for the residual errors. No overall dimensional check is available for the installed BBIII, therefore no further compensation of their effects is thinkable. Another important source of error revealed to be a non linear response of some Hall probes with the current, which seemed to be due to an intrinsic nonlinearity of the 
probe at low field. This effect is somewhat masked for the probes that measure the large signals (e.g., the tangential field probes), but it is not negligible for some of the radial field probes that measure the smallest signals. This worsens not only the accuracy of the signals measured at $77 \mathrm{~K}$, but also any calculation of the contribution of the transport current to the probe signal, performed using the signals taken @ $77 \mathrm{~K}$ as calibration constants. For these reasons, we can assess that, on the basis of the data available, the code can reproduce the signals due to a uniform current distribution among the strands within the measurement error.

\section{AnAlysis of the PUlses at LOW Temperature}

The behavior of BBIII at low temperature has been investigated as a function of time for pulses CDM3\040624\_1449\_4K\_70kA (pulse 1449) and CDM3 $\_40623 \backslash 1600 \backslash 4 K \backslash 70 k A$ (pulse 1600), in which a trapezoidal current waveform with a plateau value of $70 \mathrm{kA}$ was impressed. These pulses had the same overall shape, but different current ramp-up and ramp-down rates $(10 \mathrm{kA} / \mathrm{s}$ in pulse 1449 , and $100 \mathrm{~A} / \mathrm{s}$ in pulse 1600), which led to quite different transient behaviors. In both pulses, no heating was applied and the input helium temperature was kept constant @ $4.55 \mathrm{~K}$. No strand resistive transition occurred, so that these pulses are suitable to check the THELMA capability to reproduce the measured magnetic field when the strand currents are driven mainly by their inductances and their contact resistances with the joints. In fact, the inter-petal resistance is very high in BBIII, being the petals wrapped with stainless steel. In the analysis, pulse 1449 was used to tune the model parameters, which have then been used to simulate pulse 1600 .

\section{A. Results From the Model With 6 Macrostrands}

In the model with 6 macrostrands, for a given geometry, the cable behavior is determined by the distributed contact resistance $R_{p t_{j}}$ between petals and resistive saddle, which is not known. However, its value can be indirectly determined fitting the measured and the computed exponential decay main time constants of the field signals during the plateau. From pulse 1449 , the optimal $R_{p t_{j}}$ was found to be $0.15 \mathrm{n} \Omega \cdot \mathrm{m}$, which corresponds to the total BBIII resistance (at the model power supply leads), $R_{\text {tot }}=1.05 \mathrm{n} \Omega$. This value includes the saddles of both the joints and their resistive contacts with the petals, and it is consistent with the measured joint resistances $(0.7 \mathrm{n} \Omega$ per joint @ $70 \mathrm{kA}$, measured between the two joined conductors). The measured and the computed main time constants are about $575 \mathrm{~s}$. The contribution of the bulk material of the resistive saddles to $R_{\text {tot }}$ is only $37 \%$, which means that the optimization of $R_{p t_{j}}$ is well conditioned. Since, in BBIII joints, the cable wrappings and strand surface coatings have been removed in the contact area with the saddle, the optimal $R_{p t_{j}}$ value could be compared with the inter-petal contact resistance $R_{c_{I B}}$ measured for ITER-relevant CICC without petal wrappings and bare strand (see, for instance, the results of CRPP prototype reported in [5]). These values are even 2 orders of magnitude larger than $R_{p t_{j}}$, probably due to the different nature of the strand/saddle contact, and to the much stronger cable compaction in the joint.

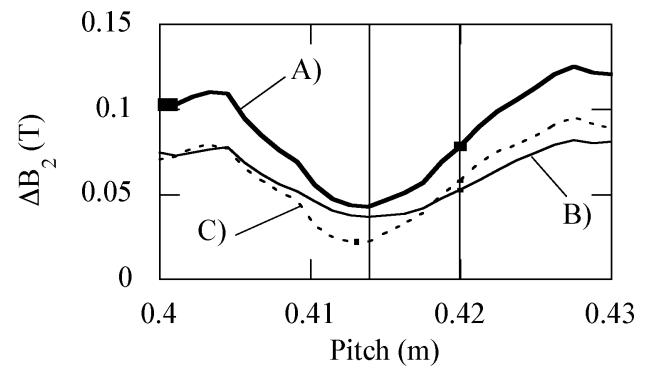

Fig. 4. Pulse 1449: Norm 2 of the difference $\Delta \mathbf{B}$ between measured and computed signals as a function of the twist pitch, at the end of the plateau @ $70 \mathrm{kA}$. (A) all HP5 and HP6 probes. (B) HP5 and HP6 tangential field probes. (C) HP5 and HP6 radial field probes.

This means that any experimental data about the contact resistances in the joint zones can be obtained only from joint prototypes and cannot be extrapolated from the existing data for CICCs.

As a second step, assuming the optimized $R_{p t_{j}}$ value and the corrected probes geometry, we investigated also the effect of the petal twist pitch and the petal initial angular position, which is the angle at which the strand/macrostrand is positioned with respect to a given radial direction, at the inlet cable cross-section, The analysis of these two parameters is very important, since the manufacturing tolerance on the twist pitch is relatively wide and the initial angular position is a totally random variable in the busbar manufacturing process. The twist pitch affects the DC cable behavior in a nearly periodical way: for a given initial angular position (which determines the distribution of the contacts between petals and saddle), being the cable length much longer than the twist pitch, even a small pitch difference involves a large difference in the final angular position of the petals, thus changes in a dramatic way the pattern of their contacts with the saddle of the outlet joint. During the transient phases of the pulses at low temperature, this influence is reduced by the inductive effects, which are much less affected by small variations of the twist pitch. Fig. 4 shows the norm 2 value, $\Delta B_{2}(p)$, of array $\Delta \mathbf{B}$ of the differences between the measured and computed probe signals as a function of the twist pitch $p$. These values are considered at the end of the plateau. The figure shows that a remarkable reduction of $\Delta B_{2}$ is present for a petal twist pitch $p=413.9 \mathrm{~mm}$, which corresponds to the minimum $\Delta B_{2}$ value closest to the nominal twist pitch $(420 \mathrm{~mm})$. The figure shows also that the field differences have the same magnitude for the radial and the tangential field probes. The minimum value occurs for the same $p$ for both types of the field probes. For these reasons $p=413.9 \mathrm{~mm}$ has been adopted in the 6 macrostrand model instead of the nominal value. The computations show also that the initial angular position has a minor effect on the signal values: for different initial angular positions the whole object "cable+joints" behaves like a DC rotating dynamo, whose rotor and brushes correspond respectively to the cable and the saddles. Different angular positions correspond to a rotation of the cable around its middle line, which involves a change of current in the petal, compensated by a change in its neighbor petals, so that the magnetic field in the proximity of the cable changes only slightly. The measured and the computed radial field probe signals as a function of time are reported in Fig. 5 

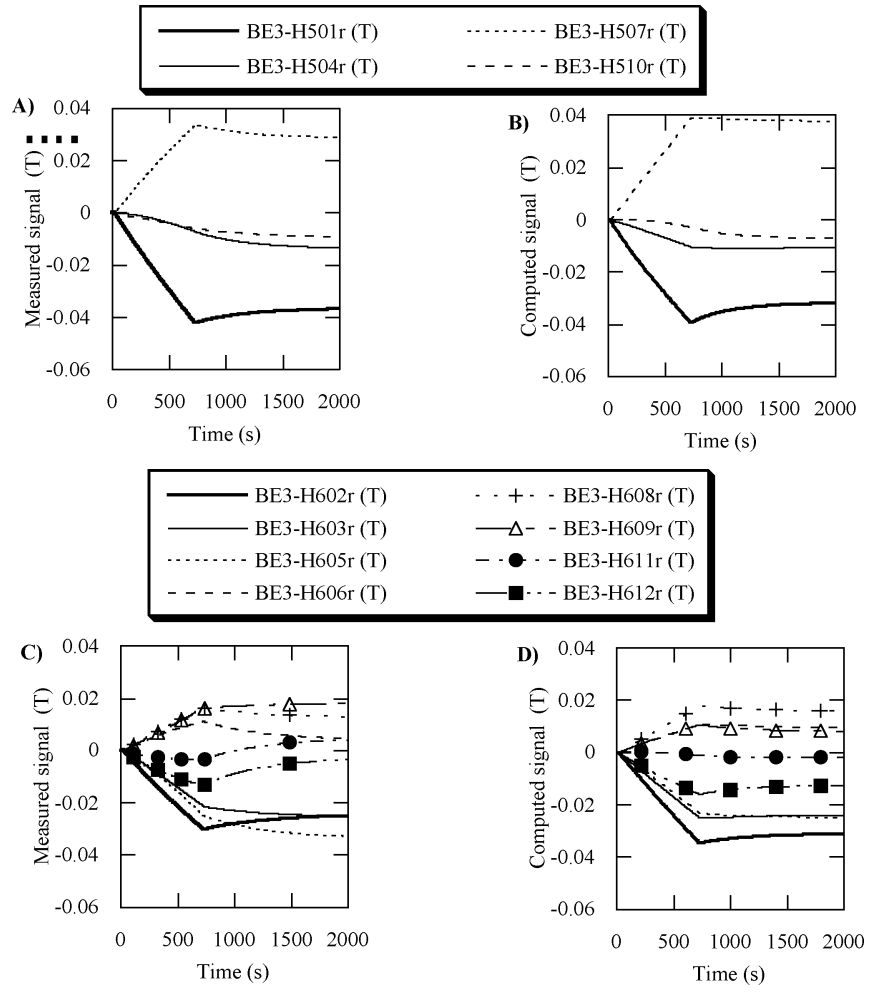

Fig. 5. Pulse 1600: measured (left plots) and computed (right plots) radial field probe signals.

for pulse 1600. The waveforms of the tangential field probe signals have not been reported, being their computed and measured waveforms nearly coincident. The comparison between these so small signals is a very severe test and an acceptable agreement means a good accuracy of the model. As can be appreciated, with this model, the computed and the measured waveforms are close for many signals, while larger errors are present for the smallest signals. Most of such error corresponds to the residual field error still present after the probe location and alignment correction (Fig. 2, described above).

At any time, the computed currents are almost uniform along each macrostrand, due to the relatively high contact resistance of the petal wrappings, and show a slightly delayed linear increase during ramp-up, followed by an exponential decay toward the DC value. The maximum current unbalance $\left(I_{\max }-\right.$ $I_{\text {ave }}$ ) among the macrostrands ranges from $7 \%$ at the end of the ramp-up, up to $9 \%$ at the end of the plateau. The effect of the initial angular position corresponds to further $\pm 1.5 \%$. These percentage values are referred to the ideal plateau macrostrand current, $I_{\text {ave }}=11.66 \mathrm{kA}$, corresponding to a uniformly distributed transport current of $70 \mathrm{kA}$.

The results presented above are to be taken into account when identification of the petal currents is performed starting from the measured probe signals. In fact, to solve this inverse problem, a high accuracy of the field computation is required to guarantee meaningful results [10]. In BBIII, in the absence of a resistive transition, the entity of the DC petal current unbalances is so low that the field error is not negligible, and the identified current waveforms may have only a qualitative meaning. However,

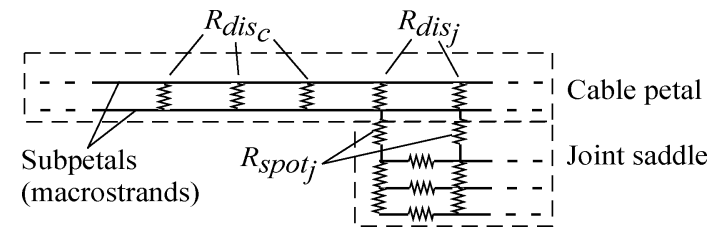

Fig. 6. Model with 24 macrostrands: detail of the equivalent lumped network of the joint, showing the inter-subpetal and the spot contact resistances between the subpetal and the saddle.

preliminary results of the application of the current identification to $T_{c s}$ pulses of BBIII show that, just before the transition, the entity of the current unbalances is so large that a low identification error can be reached, with meaningful identified current waveforms.

\section{B. Results From the Model With 24 Macrostrands}

The model with 24 macrostrands has been used to evaluate the effect of a more detailed cable model on the field and the current distribution. Assuming that negligible conductances are present between the subpetals of different petals, three unknown resistance values had to be tuned with this model: the cable inter-subpetal distributed contact resistance outside and inside the joint, $R_{\mathrm{dis}_{\mathrm{c}}}$ and $R_{\mathrm{dis}_{\mathrm{j}}}$, and the spot contact resistance between the subpetal and the saddle, $R_{\text {spot }}$ (Fig. 6). Unfortunately, for BBIII, the only available data are the joint resistance and the measured field waveforms, therefore some additional hypotheses have been made starting from the results of simulations of other similar CICC samples, from geometrical considerations and experimental results [11]. For this reason, the results of this model cannot be considered as a code validation, nevertheless they confirm the stability of the results when the model becomes more detailed and show a smooth model behavior as a function of its characteristic electrical and geometrical parameters. The adopted contact resistances are $R_{\mathrm{dis}_{\mathrm{c}}}=14.4 \mathrm{n} \Omega \cdot \mathrm{m}$, $R_{\mathrm{dis}_{\mathrm{j}}}=0.144 \mathrm{n} \Omega \cdot \mathrm{m}$ and $R_{\mathrm{spot}_{\mathrm{j}}}=7.22 \mathrm{n} \Omega$. With this model, we analyzed the DC behavior @ $70 \mathrm{kA}$, and pulse 1449 as a function of time. In both cases, the computed probe signals are close to those obtained with the 6 macrostrands model but do not show a remarkable improvement, probably because no more accurate tuning of the model parameters (resistances, geometrical data) could be carried out with the data available.

The computed current distribution along the macrostrands is quite uneven both along and among the subpetals, and shows dramatic transient current redistributions inside and close to the cable joints, due to the uneven subpetal-saddle contact distribution and to the inductive parameters. When the DC regime is reached, in a very long plateau, the subpetal currents have a piecewise linear distributions along the cable, since each petal is perfectly equipotential (Fig. 7). Fig. 8 compares the petal currents in DC @ $70 \mathrm{kA}$ resulting from the 24 macrostrands model with those obtained from the 6 macrostrands model: despite the large differences between the macrostrand currents, the resulting petal currents are quite close in the two models: the differences are due to different bundles angular initial values, 

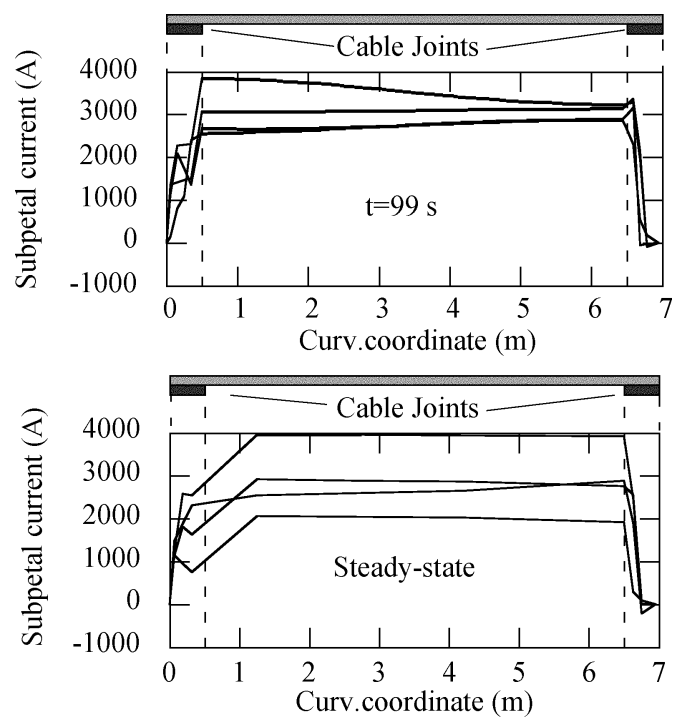

Fig. 7. Pulse 1449 , model with 24 subpetals, petal \# 1 . Above: computed subpetal currents after $92 \mathrm{~s}$ of plateau $(t=99 \mathrm{~s})$. Below: the same currents in DC at the plateau current.

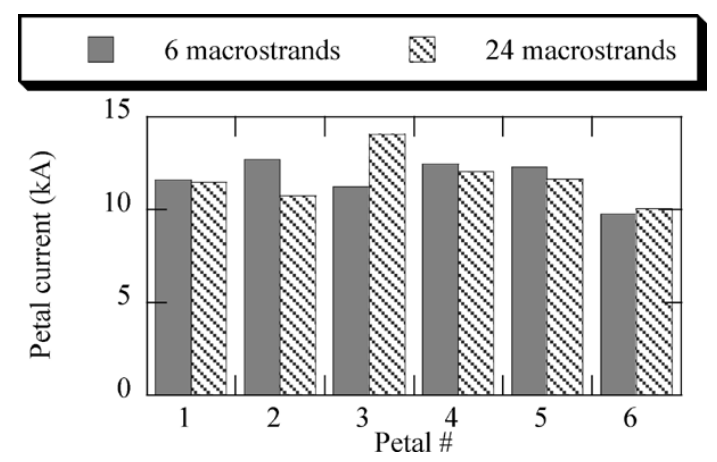

Fig. 8. DC analysis @ $70 \mathrm{kA}$ : comparison between the petal currents from the 6 and the 24 macrostrand models.

which give rise to different patterns of contacts between the macrostrands and the joint saddles.

\section{CONCLUSION}

A first validation of the THELMA electromagnetic model against the experimental data taken on BBIII has been presented. For the majority of the Hall probes considered, the computed calibration constants are very close to the experimental ones, with an error in any case below the uncertainty of the measurements. The simulation of pulses in transient regime and in absence of resistive transition has given magnetic field waveforms close to the measured ones, even using a relatively simple modelization of the cable cross-section. The analyses have shown that the simulation of the magnetic field probe signals is a critical item, requiring an accurate knowledge of the shape of the object under test, as well as an accurate calibration of the magnetic field probes. On the other hand, other output data of the CICC electromagnetic model, like the current unbalances among the strands and the dissipated power, are much less dependent on these uncertainties, therefore reliable results can be obtained also for much complex objects, like for instance the ITER Poloidal Field Conductor Insert. The analyses have evidenced also the need of a large experimental database, mostly about the contact resistances in joints and terminations.

THELMA is now going to be improved from the point of view of both the computation speed and the model capability to describe bigger and more complex objects like the next CICC prototypes, which are going to be simulated.

\section{REFERENCES}

[1] D. Ciazynski and J. L. Duchateau, "Validation of the CEA electrical network model for the ITER coils," IEEE Trans. Appl. Supercond., vol. 11, no. 1, pp. 1530-1533, Mar. 2000.

[2] A. Nijhuis et al., "Model study on AC loss and current distribution in a superconducting multi strand cable," Appl. Superc. Centre, Twente University, The Netherlands, Rep. UT-NET 20 001-1, Jan. 2000.

[3] F. Bellina et al., "Superconductive cables current distribution analysis," Fusion Eng. Des., vol. 66, no. 68, pp. 1159-1163, 2003.

[4] R. Zanino et al., "Current distribution measurement on the ITER-type NbTi bus bar III," IEEE Trans. Appl. Supercond., vol. 15, no. 2, pp. 1407-1410, Jun. 2005.

[5] A. Nijhuis, Y. Ilyjn, and W. Abas, "Electromagnetic and mechanical performance of eight prototype ITER NbTi full-size CICC's under transverse loading up to 40000 cycles," Appl. Superc. Centre, Twente University, The Netherlands, Final Report, Tasks 3, 4, 5 and 6, Contract EFDA-99-502, Mar. 2003.

[6] F. Bellina, P. Bettini, and F. Trevisan, "Electromagnetic analysis of superconducting cables and joints in transient regime," IEEE Trans. Appl. Supercond., vol. 14, no. 2, pp. 1356-1359, Jun. 2004.

[7] P. L. Ribani, "CDCABLE: A code to calculate current distribution in superconducting multi-filamentary cables," TASK N. TW0-T400-1/101 Deliverable N. 8, Intermediate reports Jun., 2001, University of Bologna, Italy, May 2002.

[8] M. Breschi, M. Ciotti, and P. L. Ribani, "Analysis of inductance coefficients in multistrand cables: analytical, numerical and experimental results," IEEE Trans. Appl. Supercond., vol. 15, no. 3, pp. 3797-3807, Sep. 2005.

[9] M. Polak, "BB-III experiment: construction, testing and installation of hall probe devices and installation of thermometers," Bratislava, Slovakia, Institute of Electrical Engineering, Slovak Academy of Sciences, Nov. 2003, unpublished.

[10] F. Bellina, "Current distribution identification in PF-FSJS," presented at the MT-19 Conf., Genoa, Italy, Sep. 2005, Paper TUA04PO06, unpublished.

[11] — "THELMA analyses of cables and joints," DIEGM Tech. Rep, Udine University, Italy, 2005. 